\title{
The effectiveness of sphenopalatine ganglion blockade in chronic migraine resistant to medical treatment
}

\author{
${ }^{1,2}$ Nermin Tepe $M D,{ }^{3}$ Oktay Faysal Tertemiz $M D$ \\ ${ }^{1}$ Department of Neurology, Faculty of Medicine, Ballkesir University, Balıkesir; ${ }^{2}$ Neuropsychiatry \\ Centre, Gazi University, Besevler, Ankara; ${ }^{3}$ Department of Anesthesiology and Algology, Health \\ Sciences University Tepecik Training And Research Hospital, İzir, Turkey
}

\begin{abstract}
Objective: To assess the effectiveness of sphenopalatine ganglion blockade (SPGB) as an alternative treatment for patients with chronic migraine resistant to medical treatment. Methods: In total, 23 patients with chronic migraine resistant to medical treatment underwent 4 sessions of bilateral transnasal SPGB bi-weekly, and the monthly number of headache days, duration, and numerical rating scale (NRS) response results were recorded. Results: Compared with before SPGB, the frequency of migraine attacks per month $(23.66 \pm 9.8 v s .10 .5 \pm 11.9$ days $)$, duration of headaches $(31.3 \pm 16.8$ $v s .18 .4 \pm 9.8 \mathrm{~h})$, and NRS score $(9 \pm 0.99 v s .5 .1 \pm 2.1)$ all improved significantly $(p<0.05)$ eight weeks after intervention.

Conclusion: Transnasal SPGB is an alternative to conventional therapy because it benefits patients with chronic migraine resistant to medical treatment, is easy to administer, and has few side effects.
\end{abstract}

Keywords: Migraine, sphenopalatine ganglion blockade, numerical rating scale

\section{INTRODUCTION}

The beta version of the ICHD-3 defines chronic migraines as headaches that have been present for at least 3 months, occur 15 days or more in a month, and have migraine features present at least 8 days a month. ${ }^{1}$ Various factors contribute to the development of chronic migraine, including failure of symptomatic treatment, overuse of acute migraine medicines, high frequency of episodes, female gender, and low socio-economic levels. ${ }^{2}$ The main pathway in the pathophysiology of migraine is the transmission of nociceptive signals originating from the meninges to the somatosensory cortex via the trigeminal ganglion, medullar dorsal horn and thalamus. Various autonomic symptoms can occur during a migraine episode, such as photophobia, phonophobia, nausea, vomiting, and nasal congestion. ${ }^{3}$

The sphenopalatine ganglion (SPG) is an extracranial parasympathetic ganglion located in the pterygopalatine fossa, in the posterolateral middle nasal concha. It has sensory, parasympathetic, and sympathetic afferents. It is part of the trigeminal-autonomic reflex, which is involved in the pathogenesis of migraine. It is activated by stimulation of the superior salivatory nucleus by trigeminal afferent nerves. Afferent trigeminal sensory neurons originating from meningeal veins extend to the pons via the thalamus. The neurons in the pons reflexively stimulate the superior salivatory nucleus, which increases the facial nerve and parasympathetic output via the otic and carotid ganglia. ${ }^{4}$ The parasympathetic efferent nerves originating from the SPG stimulate the meningeal vessels, lacrimal glands, nasal and pharyngeal mucosa, and this pathway is called the trigeminal-autonomic reflex. The parasympathetic output from the SPG contributes to the vasodilatation of cranial blood vessels that occurs during a migraine. This causes inflammatory mediators to extravasate from blood vessels and triggers a migraine headache by activating meningeal nociceptors.

Medical treatment, botulinum toxin treatment and interventional methods are treatment options for chronic migraine. SPG block (SPGB) is an interventional method used to treat trigeminal neuralgia, persistent idiopathic facial pain, acute migraine, acute and chronic cluster headaches, as well as facial neuralgias. ${ }^{5}$ SPGB can be performed transnasally using lidocaine or bupivacaine for local anesthesia. ${ }^{6}$

Address correspondence to: Dr. Nermin Tepe, Balıkesir University Faculty of Medicine, Department of Neurology, Balıkesir, Turkey. Tel: 05057574359, Email: tepenermin@gmail.com

Date of Submission: 9 June 2021; Date of Acceptance: 3 August 2021

https://doi.org/10.54029/2021jxn 
This study was conducted to assess the effectiveness of bilateral transnasal SPGB on the frequency and severity of attacks in patients with chronic migraine resistant to medical treatment (i.e., patients taking at least three prophylactic drugs for at least 3 months and having at least 8 headache days per month). ${ }^{7}$

\section{METHODS}

After obtaining approval from the Balıkesir University Clinical Research Ethics Committee (2019/102), the records of the treatment response in patients with chronic migraine resistant to medical treatment who underwent transnasal bilateral SPGB in the Algology Clinic from February through May 2019 were reviewed retrospectively. The numerical rating scale (NRS) for the severity of headaches, monthly number of days with headaches, and duration of attacks in patients between the ages of 18 and 65 with chronic migraine resistant to medical treatment seen in our outpatient clinic were collected. Patients were excluded if they had cleft lip and palate, choanal atresia, nasal septal deformities such as atrophic rhinitis, septal perforation, history of nasal/mid-facial trauma or surgery in the previous 3 months, a bleeding disorder such as hemophilia, a history of allergies, or if they were pregnant or lactating, using anticoagulants or had primary headaches other than migraine.

After obtaining consent, each patient was placed in a supine position, bringing the neck to extension, and a transnasal bilateral SPGB was applied using cotton-tipped swabs soaked with $0.5 \% 0.5 \mathrm{~mL}$ bupivacaine for 15 minutes. Next, $0.5 \mathrm{~mL}$ of bupivacaine was dripped into each nostril from the cotton-free ends of the swabs for another 15 minutes, and the procedure was completed in 30 minutes (Figure 1). The procedure was performed every 2 weeks, for 4 sessions in total. All blocks were performed by the same physician. The frequency and duration of attacks and NRS scores before and after the procedure were recorded before the treatment and at each procedure.

\section{Statistical analysis}

Data was analyzed using the Statistical Package for the Social Sciences for Windows 21.0. Conformity with the normal distribution was checked using the Kolmogorov-Smirnov test. The chi-square test was used to compare qualitative data. Student's $t$-test was used to compare normally distributed quantitative data and the Mann-Whitney $U$-test otherwise. Repeatedmeasures analysis of variance with the post hoc Bonferroni correction was used.

\section{RESULTS}

The study population included 23 patients (21 females, 2 males). The efficacy of the intervention was assessed 8 weeks after the last injection. The average frequency of monthly episodes before SPGB was $23.6 \pm 9.8$ versus $10.5 \pm 11.9$ days afterwards; the average duration of headache before SPGB was $31.3 \pm 16.8$ versus $18.4 \pm 9.8$ hours afterwards; and the average NRS score before SPGB was $9 \pm 0.99$ versus $5.1 \pm 2.1$ afterwards. The differences in all three scores at 8 weeks were significant $(p<0.05)$ (Figure 2-4).

One patient was excluded from the study

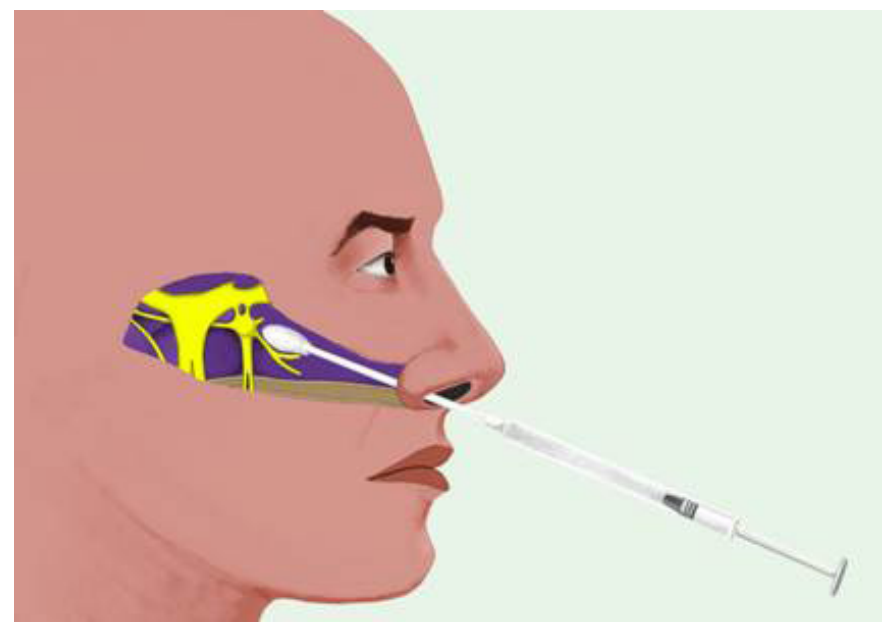

Figure 1. In the supine position, block was applied to the transnasal bilateral SPG using cotton-tipped 


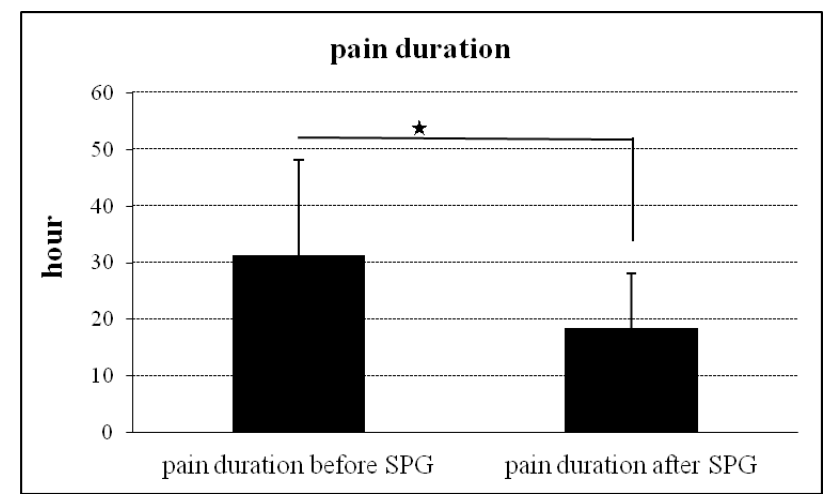

Figure 2. The mean duration of attacks after SPG blockage significantly decreased compared to before treatment. $* \mathrm{p}<0.05$

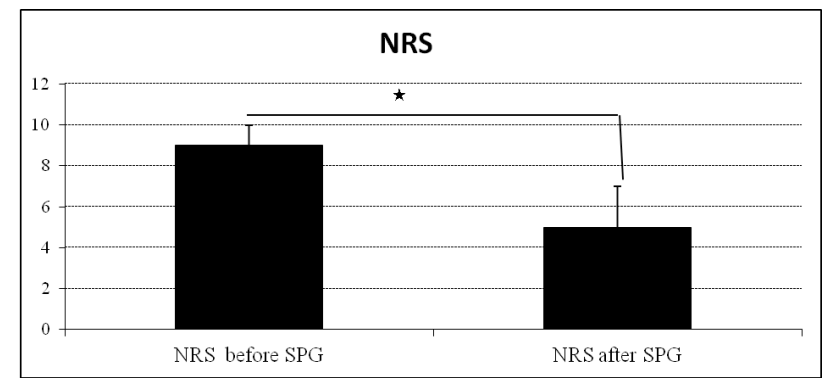

Figure 3. The mean attack severity after SPG blockage significantly decreased compared to before treatment. NRS: Numeric Rating Scale, SPG: phenopalatine ganglion block. $* \mathrm{p}<0.05$

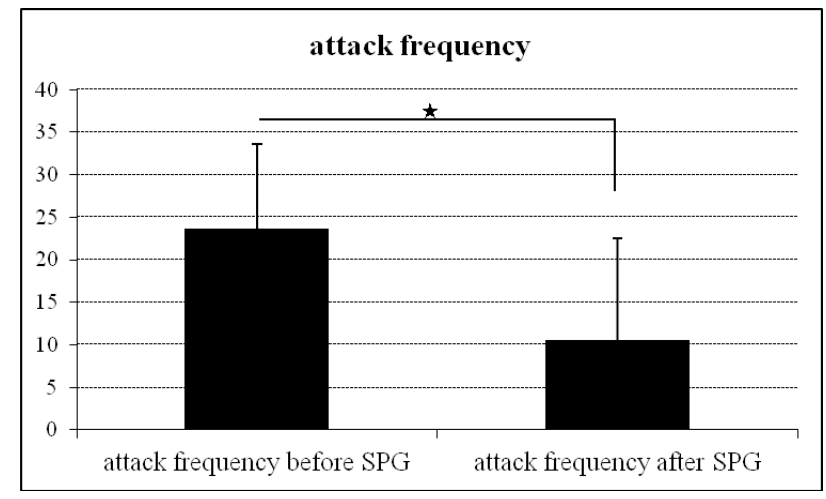

Figure 4. The mean monthly attack frequency after SPG blockage decreased statistically significantly compared to before treatment $* \mathrm{p}<0.05$.

when severe vomiting and vertigo requiring symptomatic treatment occurred during the first procedure. No other serious side effects were observed in patients. Minor complications included minimal nasal bleeding, sneezing, coughing, and discomfort.

\section{DISCUSSION}

This study was conducted to evaluate the effectiveness of transnasal SPGB in patients with chronic migraine resistant to medical treatment. Significant decreases were observed in the monthly frequency of attacks, duration of attacks, and NRS scores in patients who underwent SPGB. Therefore, transnasal SPGB is a treatment option 
in patients with resistant chronic migraine due to its ease of administration and absence of severe side effects or complications.

Previous evidence supports the use of SPGB, radiofrequency ablation or SPG neurostimulation in trigeminal autonomic cephalgia accompanied by autonomic findings, mainly in cluster headache. ${ }^{8}$ Smell, nutrition, insomnia and stress factors, which can trigger migraine headaches, affect various regions of the brain and cause activation of the sphenopalatine ganglion (SPG) by stimulating the superior salivatory nucleus. ${ }^{9}$ Repeated SPGB disrupts the autonomic headache cycle. Modulating the afferent sensory fibers of the caudal trigeminal nucleus using SPGB may alter the processing of pain, alleviating it. ${ }^{10}$ Interventional methods can improve the quality of daily life in patients with chronic migraine resistant to medical treatment. No previous studies have assessed SPGB as a treatment option for migraine patients not responding to medical treatment, so we examined this option. We performed blockade every 2 weeks for prophylactic purposes, regardless of the timing of attacks. Some authors have performed SPGB in migraine patients at the time of attack, and the few studies investigating SPGB for chronic migraine were mostly conducted during episodes. In a recent study, transnasal SPGB with $0.3 \mathrm{~mL}$ $0.5 \%$ bupivacaine or saline was administered twice a week for 6 weeks during an acute episode using double-blind placebo-controlled randomization in patients with chronic migraine. The authors found a significant decrease in the NRS pain score compared to the placebo group; with repeated SPGB, the duration of migraine episodes decreased for 6 weeks, although the change was not significant. ${ }^{11}$ In another study, $0.4 \mathrm{~mL}$ of $4 \%$ lidocaine was dripped into the nostrils of 23 patients with migraines during an episode; resulting in the severity of the episode decreasing significantly in 12 of 23 patients. ${ }^{12}$ Most previous studies involved the use of a Tx360 Nasal Applicator. We used a drug-soaked cotton swab, so that the costs of the procedure would be lower and the drug would remain longer in the area. Furthermore, the dosage of the drug we administered was higher, which might have increased the effectiveness of the block. The monthly frequency of headaches, duration of episodes and NRS scores at 8 weeks were all decreased significantly compared to before the treatment. Despite the higher dosage of anesthetic, we did not observe any severe complications. However, the optimal minimum and maximum number of blocks has not been established and there is no consensus in the literature on the dosage of drugs to use.

In one study, suprazygomatic SPGB was performed using $5 \mathrm{~mL}$ of a mixture of dexamethasone $(1 \mathrm{~mL})$ and $0.5 \%$ ropivacaine $(4 \mathrm{~mL})$ in status migrainosus, and the headache severity decreased after 30 minutes. ${ }^{13}$ In another study, 10 patients with resistant chronic migraine were treated with $25 \mathrm{IU}$ of botulinum toxin A at the SPGB bilaterally. The authors observed at least a $50 \%$ reduction in the number of days with moderate to severe headache in 8 of 10 patients at 3-month follow-up, but they used the percutaneous infrazygomatic approach instead of a transnasal technique..$^{14}$ The side effects of local anesthetics include orthostatic hypotension, nasal bleeding, and systemic toxicity of local anesthetics. In the current study, SPGB was discontinued when one patient experienced vertigo and vomiting; no other side effects were observed in any of the 23 patients included in the study.

Although the SPG plays an integral role in the pathophysiology of facial pain and trigeminal autonomic cephalgia, its exact role in the formation and maintenance of migraine remains unclear. SPGB is potentially effective for the relief of headaches in chronic migraine. Although we found SPGB to be effective in patients with chronic migraine resistant to medical treatment, the long-term results and the ideal initial number of sessions are not yet known. Transnasal SPGB deserves to be used more widely in clinical practice because it is a simple, relatively low-risk, inexpensive procedure. It would decrease the number of hospitalized patients, cost of treatment per patient, as well as the frequency and severity of episodes, while improving the patient's quality of life. Therefore, large randomized double-blind placebo-responsive studies of its efficacy are required.

The limitations of the present study are the small study population, retrospective assessment, and the fact that it did not have a randomized controlled design.

\section{DISCLOSURE}

Conflict of interest: None

\section{REFERENCES}

1. Headache Classification Subcommittee of the International Headache Society. The international classification of headache disorders: 3rd edition (beta version). Cephalalgia 2013;33:629-808. 
2. Katsarava Z, Buse DC, Manack AN, et al. Defining the differences between episodic migraine and chronic migraine. Curr Pain Headache Rep 2012;16:86-92.

3. Goadsby PJ, Holland PR, Martins-Oliviera M, et al. Pathophysiology of migraine: A disorder of sensory processing. Physiol Rev 2017;97:553-622.

4. Goadsby PJ, Lipton RB, Ferrari MD. Migrainecurrent understanding and treatment. $N$ Engl J Med 2002; 346:257-70.

5. Piagkou M, Demesticha T, Troupis $\mathrm{T}$, et al. The pterygopalatine ganglion and its role in various pain syndromes: from anatomy to clinical practice. Pain Practice 2012;12:399-412.

6. Cady RK, Saper J, Dexter K, et al. Long-term efficacy of a double-blind, placebo-controlled, randomized study for repetitive sphenopalatine blockade with bupivacaine vs saline with the Tx $360 \AA$ device for treatment of chronic migraine. Headache 2015;55:529-42.

7. Sacco S, Braschinsky M, Ducros A, et al. European headache federation consensus on the definition of resistant and refractory migraine. J Headache Pain 2020;21:76.

8. Ho KWD, Przkora R, Kumar S. Sphenopalatine ganglion: block, radiofrequency ablation and neurostimulation - a systematic review. J Headache Pain 2017;18:118.

9. Piagkou M, Demesticha T, Troupis $\mathrm{T}$, et al. The pterygopalatine ganglion and its role in various pain syndromes: from anatomy to clinical practice. Pain Pract 2012;12:399-412.

10. Candido KD, Massey ST, Sauer R, et al. A novel revision to the classical transnasal topical sphenopalatine ganglion block for the treatment of headache and facial pain. Pain Physician 2013;16:769-78.

11. Cady RK, Saper J, Dexter K, et al. Long-term efficacy of a double-blind, placebo-controlled, randomized study for repetitive sphenopalatine blockade with bupivacaine vs. saline with the Tx360 device for treatment of chronic migraine. Headache 2015;55:529-42.

12. Kudrow L, Kudrow DB, Sandweiss JH. Rapid and sustained relief of migraine attacks with intranasal lidocaine: preliminary findings. Headache: $\mathrm{J} \mathrm{Head}$ Face Pain 1995;35:79-82.

13. Mehta D, Leary MC, Yacoub HA, et al. The effect of regional anesthetic sphenopalatine ganglion block on self-reported pain in patients with status migrainosus. Headache 2019;59:69-76.

14. Bratbak DF, Nordgård S, Stovner LJ, et al. Pilot study of sphenopalatine injection of onabotulinum toxin A for the treatment of intractable chronic migraine. Cephalalgia 2017;37: 356-64. 